\title{
NECESIDAD SOCIAL DE LA INTERVENCIÓN UNIVERSITARIA EN LOS PROCESOS MEDIOAMBIENTALES EN LAS COMUNIDADES COSTERAS DE ANGOLA.
}

\author{
Joao Domingos Victor
}

Centro de Estudios de la Educación. República de Cuba.

\section{Lizette de la Concepción Pérez Martínez}

Centro de Estudios de la Educación. Universidad de Oriente. República de Cuba.

Santa Nurkis Díaz Rodríguez

Delegación Territorial del Ministerio de Ciencias

Tecnología y Medio Ambiente. República de Cuba

Alcides Francisco Antúnez Sánchez

Facultad de Ciencias Sociales y Humanísticas

Universidad de Granma. Republica de Cuba Necesidad social de la intervención universitaria en los procesos medioambientales en las comunidades costeras de Angola

\author{
http://dx.doi.org/10.5209/rev_NOMA.2014.v42.n2.48775
}

Resumen.- El trabajo tiene como pretensión fundamental realizar un estudio gnoseológico sobre el estado de conocimiento del Derecho del Medio Ambiente en la República de Angola, para esto se realiza un estudio y análisis de la actual normativa ambiental vigente y de los sistemas de derechos utilizados en materia de derecho comparado.

Se ejecuta además todo un estudio desde las ciencias sociales que permiten hacer un abordaje desde una dimensión social a la problema ambiental, principales factores y adversos ambientales, que inciden en la protección de los recursos ambientales en Angola.

Se realiza un estudio de la función universitaria como generadora de cultura, en el caso específico de su encargo para sustanciar los procesos ambientales, se hace imprescindible dirigir sus procesos, no solo en el sentido de hacer coincidir los objetivos de la práctica y la profesión con la conducta del profesional, sino a la formación general e integral del ciudadano como gestor de esos procesos, considerando un estilo de pensamiento científico.

Para esto se identifica que la formación en el estudiante, particularmente de la carrera de Derecho, de un estilo de pensamiento desde su profesión, que trascienda sus límites y que el autor del presente trabajo advierte como un estilo de pensamiento científico totalizador; el ambiental. Utilizando para esto como métodos científicos de apoyo a la investigación el histórico lógico, el exegético-jurídico, el análisis y revisión de documentos científicos tanto sociales como jurídicos.

Palabras claves: educación, cultura, derecho ambiental, responsabilidad ambiental, intervención universitaria.

Abstract.- The work has like fundamental hope to accomplish a study gnoseológico on the status of knowledge of the right of The Ambient midway at the Republic of Angola, ad hoc a study and analysis of the present-day environmental ground rules in use of the systems of rights utilized on the subject of comparative jurisprudence and are accomplished.

It executes besides all of a study from the social sciences that they enable doing a boarding from a social dimension to her environmental problem, principal factors and adverse environmental, that they have an effect on the protection of the environmental resources in Angola.

A study of the university show like generating plant of culture, in the specific case of his assignment to substantiate the environmental processes, to direct his processes becomes essential, comes true you did not sole in the sense to match up the objectives of practice and 
the profession with the professional's conduct, but to the general and integral formation of the citizen like manager of those processes considering a style of scientific thought.

Ad hoc you provide evidence of identity than the formation in the student, particularly of the race by right, of a style of thought from his profession, that you transcend his limits and that the author of the present work notices like a style of scientific totalizing thought; The environmental. Utilizing the historic logician to the investigation ad hoc like scientific backup methods, the exegetic juridical, analysis and revision of scientific documents so much social I eat juridical.

Key words: educación, cultura, derecho ambiental, responsabilidad ambiental.

\section{INTRODUCCIÓN}

El desarrollo del hombre en sociedad a partir de su intervención en procesos sociales determina la relación hombre-naturaleza-sociedad por lo que cualquier análisis posterior debe estar signado por el reconocimiento de esa relación y la percepción del hombre como un sujeto cognoscente, consciente de su accionar en esos procesos sociales.

Aún cuando el fraccionamiento del conocimiento científico es una realidad ineludible, la única vía de solución a los problemas ambientales es darle un carácter tridimensional, pues son al mismo tiempo procesos científicos, tecnológicos y ambientales.

La relación hombre-naturaleza, aunque incluye la explotación y manejo de recursos naturales, contiene varias esferas específicas de protección; que se encuentran diseminadas en toda manifestación de la conducta humana, a partir de que el hombre se desarrolla, actúa y transforma su contexto social y natural. De ahí que toda manifestación de conducta humana en sus relaciones sociales es susceptible de ocasionar un impacto negativo o positivo en el Medio Ambiente, natural o construido según sea el caso.

Consecuentemente, dada la función universitaria de generar cultura, en el caso específico de su encargo para sustanciar procesos ambientales, se hace imprescindible dirigir sus procesos, no solo en el sentido de hacer coincidir los objetivos de la práctica y la profesión con la conducta del profesional, sino a la formación general e integral del ciudadano como gestor de esos procesos, considerando un estilo de pensamiento científico.

Sopeña (2010); Chivas (2010); Díaz (2011), abordaron la formación de un estilo de pensamiento científico para determinadas ciencias. No obstante, en casos complejos, obedeciendo a causas múltiples, su solución requiere un carácter global, con el esfuerzo coordinado y conjunto de una pluralidad de actores, entre ellos, los de la educación, dado por la dinamización de su enfoque para desaislar las disciplinas que lo contemplan, y articular los actores que intervienen. Entonces, trasciende los límites de una ciencia particular desde la labor extensionista universitaria, sustentada en la educación popular.

Coincidentemente, Vega (2011), en ellos incluye "El problema Medioambiental, porque ha sido abordado de una manera fragmentada, en el conocimiento, 
primando en la sociedad de hoy el desentendimiento al respecto, no obstante, es un problema complejo"1i.

Para el profesional del Derecho la responsabilidad ambiental es un criterio de imputabilidad y no un valor que se construye como parte del entramado de relaciones sociales en las que interviene el ser humano como sujeto Díaz Rodríguez Santa Nurkis (2011).

Se impone, la formación en el estudiante, particularmente de la carrera de Derecho, de un estilo de pensamiento desde su profesión, que trascienda sus límites y que el autor del presente trabajo advierte como un estilo de pensamiento científico totalizador; el ambiental. Categoría que designa aquella función intelectual cosmovisivo sobre los procesos ambientales que entrañan una responsabilidad global más allá de los límites espacio- tiempo, es decir, una responsabilidad tanto con las generaciones futuras como con el planeta en su integridad física Victor Joao Domingos (2012).

Sirve de ejemplo, la zona costera angoleña, donde dicha responsabilidad, está limitada, caracterizándose por una deteriorada gobernabilidad ambiental, dada por la pesca ilícita, asociada a otros problemas: la pérdida de la diversidad biológica, revelada por la captura de especies cuya fragilidad está acreditada, contaminación de las aguas, degradación del suelo marino y del medio acuático. Esta falta de gobernabilidad ambiental, está determinada por los siguientes factores:

- Déficit de control y fiscalización gubernamental.

- Insuficiencias en la cantidad y calidad de embarcaciones modernas y apropiadas para la labor de fiscalización de la zona costera, matizado por obsolescencia de las ya existentes.

- Insuficiente cantidad y calidad de los Recursos Humanos que deben acometer el manejo, control y fiscalización de los recursos naturales, históricos y culturales del ambiente en Angola.

Visto así, esta valoración causal advertida desde las ciencias sociales, solo puede solventarse trabajando, a partir de la ingeniería o reingeniería de los procesos, en los que se forman los Recursos Humanos que deben acometer el manejo, control y fiscalización de los recursos naturales, históricos y culturales de las zona costeras del ambiente angolano.

La contradicción está dada a partir de la relación que se establece entre la orientación de los procesos ambientales en el Proceso Docente Educativo y la comprensión del carácter globalizante de estos procesos.

Por lo que se declara como problema las Insuficiencias en el cumplimiento de la misión universitaria de la asignatura Derecho Ambiental desde la carrera de Derecho, en la formación cultural de segmentos de la población angoleña residentes en las zonas costeras. Tomando como sector interviniente: la

\footnotetext{
${ }^{1}$ Yoandy Vega Peña, Investigador del Ministerio de Ciencia, Tecnología y Medio Ambiente de Cuba.
} 
comunidad, por ser el medio natural donde se concretan dichos procesos ambientales.

El objetivo está centrado en argumentar la necesidad social de la elaboración de una estrategia de intervención para solventar las insuficiencias en la comprensión del carácter globalizante de los procesos ambientales, a partir de la formación de un estilo de pensamiento científico, cuya dinámica parte de la labor del estudiante de la carrera de Derecho en su extensión universitaria, hacia las comunidades costeras.

Que tiene como significación Social la elaboración de una estrategia de intervención para la participación del sujeto aprehendiente de la carrera de Derecho contribuirá a la búsqueda y análisis reflexivo del conocimiento ambiental, favoreciéndose la capacidad de motivación, la orientación del docente que dirige el proceso e incluso las propias habilidades de éste para la reflexión, organizada en un sistema de acciones y medios didácticos destinados a la extensión universitaria del estudiante en las comunidades costeras.

1.- Panorámica acerca del desarrollo de la ciencia y las tecnologías: La Revolución científico técnica es el hecho que señala el punto de partida de desarrollo de la ciencia y las tecnologías, en el orden; político, social, cultural, económico e ideológico, iniciado entre los siglos XVI y XVII, cuyas consecuencias se manifiestan de diversas formas en todos los países; desarrollados, en desarrollo, subdesarrollados y en los diferentes sistemas sociales.

Esta nueva era es una era de ciencia, cada día se hace más urgente destruir los límites de la profesión entre los científicos. El científico no solo debe recurrir al descubrimiento del hecho científico, sino que debe ocupar su hacer en la gestión de procesos a fin de perfeccionar la aplicación de la ciencia desde una perspectiva globalizante.

Los procesos de enseñanza, son procesos de ciencia, entendida esta no como ente diferente de la tecnología sino como término que lo contiene, como procedimiento, sistematización, trabajo científico que se usa para entender y aplicar la ciencia y que no pierde en momento alguno, su esencia y rigor.

En consecuencia, es medular, entender el desarrollo social como un proceso complejo de implicaciones económicas, jurídicas y ambientales como regularidad esencial.

Para el logro del objetivo propuesto, se considera necesario abordar la dimensión social de la ciencia, partiendo de su conceptualización, que contribuirá a la argumentación de la necesidad social de la intervención Universitaria en los procesos medioambientales en las comunidades costeras de Angola.

La palabra ciencia, deriva etimológicamente en las lenguas modernas del vocablo latino "Scientia", que viene de "scire" que significa saber. En general, en sentido amplio y significa: conocimiento, práctica, doctrina, erudición, 
conocer, tener noticia de, estar informado. Algunos científicos encabezados por Kant, I. (1724-1804), [Apud] Rosental, M. y P.Iudin (1981:256-257), consideran la ciencia como un sistema de conocimientos, con un objetivo fundamental: descubrir las leyes de los fenómenos y encontrarles una explicación.

Esta definición analiza la ciencia unilateralmente, de manera cientificista, con finalidad limitada, sin establecer nexos sociales, ni su repercusión en la sociedad. Establece solo la relación ciencia-fenómeno, sin considerar la relación ciencia-sociedad, donde la ciencia funciona como información acumulada a lo largo del desarrollo humano, al transmitirse los descubrimientos del hombre de una época a otra de manera espiritual.

De acuerdo con el criterio de Kedrov B. (1982), la ciencia es un importantísimo elemento de la cultura espiritual, la forma superior de los conocimientos humanos, un sistema de conocimientos en desarrollo, los cuales se obtienen mediante los métodos cognoscitivos y se reflejan en conceptos exactos, cuya veracidad se demuestra a través de la práctica social. Añade además, que ciencia es un sistema de conceptos acerca de los fenómenos y leyes del mundo exterior o de la actividad espiritual de los hombres que permite prever y transformar la realidad en beneficio de la sociedad.

En este sentido el ordenamiento jurídico angolano prevé en la Constitución de la República: artículo 39 referido al Derecho al ambiente, que todos tienen derecho a vivir en un ambiente sano y no contaminado, y asimismo el deber de defenderlo y preservarlo. Asimismo en sus apartados dispone:

2. El Estado adoptará las medidas necesarias para la protección del medio ambiente y de las especies de la flora y de la fauna en todo el territorio nacional, para el mantenimiento del equilibrio ecológico, la correcta localización de las actividades económicas y la explotación y utilización racional de todos los recursos naturales, en el marco de un desarrollo sostenible y de respeto por los derechos de las generaciones futuras y de la preservación de las diferentes especies.

3. La ley castigará los actos que pongan en peligro o perjudiquen la preservación del medio ambiente.

Este derecho constitucional debe ser advertido en relación con los artículos 16 y 21 del propio cuerpo legal referidos a: Recursos naturales y Funciones fundamentales del Estado, respectivamente.

En esta mirada, la ciencia se nos presenta como una institución social; como un sistema de organización científica, cuya estructura se encuentra estrechamente vinculada con la economía, la política, los fenómenos culturales, con las necesidades y las posibilidades de la sociedad dada. En esta concepción se comprende la ciencia como un sistema de conocimientos en desarrollo, lo que supone la aplicación sistemática de métodos, formulación de problemas científicos, incorporación de teorías, renovación de sus categorías intrínsecas y su confrontación permanente con los hechos en la sociedad. 
Otra mirada esta centrada en, la necesidad es una categoría filosófica que se desprende de la esencia interna de los fenómenos, designa la ley, el orden y la estructura de los mismos. Es lo que debe ocurrir obligatoriamente en unas condiciones dadas y se complementa indefectiblemente con la casualidad (Rosental 1973:334).

Los problemas ambientales no escapan a los efectos de la relación necesidadcasualidad, consiguientemente, el hombre necesita minimizar los efectos de los impactos negativos al medio ambiente y no, dejarlo a la espontaneidad ni a la casualidad sino dirigir la sociedad del conocimiento.

En ese sentido, para lo que requiere del esfuerzo mancomunado de los seres humanos, necesitándose imperiosamente una nueva revolución científica que democratice el conocimiento, una alfabetización científica.

La democratización de los procesos sociales pasa necesariamente por el subproceso de democratización del acceso al conocimiento científico. Núñez Jover $^{2}$ (2003) cuestiona, acertadamente además, el hecho de titularnos como "la sociedad del conocimiento" sobre la idea de que información no es conocimiento y solo crea ruidos, ignorancia y desconcierto si no cuenta con un marco teórico, conceptual y axiológico.

Por otra parte, los estudios sociológicos de la educación y la ciencia de Wagle (2002), López Cerezo y Lujan (2001), Morín (1994) apuntan a la insoslayable realidad de que acceso a la universidad no significa acceso al conocimiento toda vez que ese conocimiento debe ser científico, direccionado a una intencionalidad formativa y adquirido de forma lógica y racional.

Las Ciencias, la Tecnología y la Sociedad abordan de una manera u otra el tema medioambiental, Jover (1999), (2002) (2003); Díaz Caballero (2004); Menocal (2006), advierten la imperiosa necesidad de transformar el pensamiento ambiental a partir de un proceso de educación científica que permita el equilibrio científico-ecológico.

La crisis ambiental problematiza con los paradigmas establecidos y demanda por tanto, nuevas formas de orientar los procesos de construcción del saber, que permita un análisis integral de la realidad. La cuestión ambiental actual necesita de propuestas que internalicen sobre bases ecológicas y principios jurídicos y sociales a una gestión democrática de los recursos naturales en el logro de condiciones sustentables del planeta ${ }^{3}$.

En ese sentido, se inserta esta propuesta sobre la idea además de que el conocimiento aún empírico sobre el procedimiento para desarrollar un proceso, constituye una tecnología, si es un conocimiento elaborado académicamente el que permite el desarrollo del proceso, entonces es conocimiento científico o tecnología sobre la ciencia. Advirtiendo igualmente dos niveles en el trabajo científico uno a nivel de laboratorio que deviene en el descubrimiento de un hecho científico y un nivel sobre la ciencia existente donde se trabaja la

\footnotetext{
${ }^{2}$ Dr. Jorge Núñez Jover. La ciencia y la Tecnología como procesos sociales. Habana 2003.

${ }^{3}$ Valdés Menocal, Célida. El saber Ambiental. Problemas Sociales de la Ciencia y la Tecnología. Habana 2004.
} 
ingeniería o reingeniería de procesos. Perteneciendo esta propuesta particular al segundo nivel.

2.- Caracterización de la República de Angola. Angola, matizada por ser un país situado en el suroeste de África (África Austral, conocida en época colonial Occidental), que ocupa una superficie de $1.246 .700 \mathrm{~km}^{2}$ (ver figura 1). Posee el pequeño enclave de Cabinda, localizado a $30 \mathrm{~km}$ al norte y que limita al norte y al este con la República Democrática del Congo, al este con Zambia, al sur con Namibia y al oeste con el océano Atlántico.

Tiene una costa de $1.600 \mathrm{~km}^{2}$. Compartida en siete de las 18 provincias: Cabinda, Zaire, Kuanza Sur, Bengo, Luanda, Benguela y Namibe. La costa angoleña es sumamente rica en recursos naturales, destacándose una rica diversidad biológica, grandes yacimientos minerales de petróleo, diamantes; vetas de hierro, manganeso, cobre, uranio, fosfatos y sales, entre otros; que de continuar la exploración geológica, se agregaran como descubrimientos a la lista actual.

La capital es Luanda. Su población fue estimada en 19.000.000 de habitantes hasta 2011. Es un país plurilínguístico, siendo el portugués considerado la lengua oficial para la comunicación entre los angolanos, aunque existen otras lenguas nacionales, por ejemplo: Umbundu, Kimbundu, Kikongo, Tchokwe e N'gangela (Ministerio de Educación de la República de Angola, 2003).

Fue, durante cerca de 5 siglos una colonia portuguesa, que conquistó su independencia nacional el 11 de noviembre de 1975. La Ley constitucional angolana considera la educación como un derecho de todos los ciudadanos independientemente del sexo, raza, etnia y creencia religiosa. En 1977, fue aprobado un nuevo sistema de educación cuya implementación se inició en 1978, teniendo entre sus principios generales los siguientes:

- Igualdad de oportunidades para el acceso a la continuidad de los estudios;

- Enseñanza gratuita en todos los niveles de educación;

- Perfeccionamiento constante en la preparación del personal docente.

Este sistema está constituido por una enseñanza general en base a 8 niveles (la escuela Primaria obligatoria en cuatro años), la enseñanza preuniversitario en seis semestres, la enseñanza media se realiza en cuatro años (con dos ramos, técnicos y normal) y la enseñanza superior. Según el Ministerio de la Educación de Angola (2003), en 1977, Angola disponía de cerca de 25 mil profesores pobremente formados.

El mayor impacto sociocultural del nuevo sistema de educación, lo constituyó una explosión de la matricula, con una gran afluencia de la población en edad escolar. En 1974, estudiaban cerca de medio millón de angolanos. En 1980, alcanzó 1,8 millones. Sin embargo, no fue posible mantener dichos indicadores, puesto que el país, a pesar de la independencia continúo en guerra armada, cuyas consecuencias se podían sentir principalmente en las zonas rurales. Sus efectos nocivos repercutieron profundamente en las infraestructuras escolares. Innumerables escuelas fueron destruidas. 
La guerra en Angola fue una constante desestabilización, provocando el empobrecimiento del Estado, de las poblaciones y de las escasas redes escolares. Grandes flujos de pobladores emigraban a las ciudades por considerarlas más seguras, aumentándose su concentración en ciertas provincias, como: Lubango (30\%), Benguela $(11,4 \%)$, pero principalmente Luanda (30\%) en Huíla (13\%). En las restantes provincias alcanzó un 10\%.

La situación angolana continuó, y en el año 1996, cerca del $70 \%$ de la población en edad escolar, entre 6-14 años, tuvo el riesgo de ser analfabeto, por falta de oportunidades de acceso a redes escolares. Estimándose, una tasa de analfabetismo del $60 \%$ en los mayores de 15 años. Situación social que contrasta con el 1995, en el que estimó en cerca de 4 millones de personas de ellas, 2,5 millones eran mujeres. Atenuándose la situación con la distribución de los escolares en las escuelas de las diversas provincias y creándose en la enseñanza primaria el triple turno, con 60 a 80 alumnos.

En este contexto sociocultural adverso se inician los primeros pasos en la formación de las nuevas generaciones en dicho país con miras a la preparación de la $2^{a}$ reforma del Sistema de Educación. En el año 2001 la Asamblea Nacional de la República de Angola aprobó una ley sobre la formación básica en el Sistema de Educación (Ley No. 13 de 31 de Diciembre de 2001). Este documento contenía las líneas que se pretendía emprender con el nuevo sistema, cuya estructura integra entre sus subsistemas: Educación Preescolar Enseñanza General, Enseñanza Técnico-Profesional, Formación de los Profesores, Educación de Adultos y la Enseñanza Superior.

Este nuevo proyecto de sistema de educación se preparó en condiciones muy precarias: pocos recursos, sin posibilidades de lograr la formación experimental de los estudiantes y menos, la integración de las instituciones en sus diferentes niveles con el entorno, cuestión, aún, no lograda en el país, requiriéndose trabajar en una Reforma Curricular con el fin de interrelacionar la ciencia, la tecnología, la sociedad y el ambiente en dicho contexto histórico y social, con vista al desarrollo del país, en el que se trabaja hoy con la asistencia de diversos países, entre estos se destacan los profesores cubanos.

\section{3.- Caracterización de las comunidades costeras de Angola.}

La comunidad, constituye un mecanismo intermedio entre la sociedad y el indviduo, donde se materializan todas las interacciones del sujeto, la relación sociedad -individuo, donde las personas llegan a armonizar y a establecer acciones permanentes hacia un mismo fin, para el crecimiento individual y social.

La comunidad es definida por algunos autores como: organización donde las personas se perciben como unidad social, comparten un territorio, intereses y necesidades, interactuando entre sí y promoviendo acciones colectivas a favor del crecimiento personal ysocial, llegando a manifestar sentimientos de pertenencia como expresión de su identidad comunitaria. 
La actividad fundamental es la pesca como principal fuente necesaria de alimentación y de ingresos, la población no es homogénea en sus características personales referidas a nivel de escolaridad, posibilidades económicas y reconocimiento social.

Un estudio retrospectivo de Angola, desde el censo de 1960 realizado, mostró que en la etapa colonial los grupos sociales, tenían políticamente su dependencia de Portugal y estaban compuestos por:

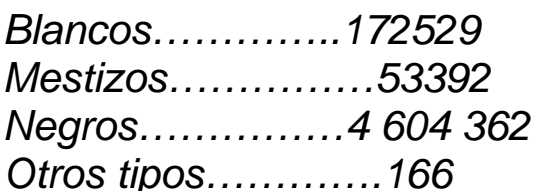

Significando que la raza negra era aproximadamente 30 veces más de negros que blancos; siendo estos últimos los que mandaban, opinaban, decidían. Un ejemplo de ello es que la alimentación ofrecida por las empresas industriales y agrícolas a sus trabajadores negros en cualquier esfera del campo de su actividad laboral, tenía un esquema de: $700 \mathrm{gr}$. de mandioca, $150 \mathrm{gr}$. de frijoles e igual cantidad de pescado seco, 100 de maní y 50 de aceite de palma.

Ello revela, carencias nutritivas, particularmente en proteínas de origen animal. Pero además aseguraban que el trabajo que el trabajo era bueno para ellos, asegurándose que de un universo de 12561 pescadores, 11193 de ellos, fueran de la raza negra, para un porcentaje de 89.1\%, mientras que 1363 , eran blancos. Predominando en aquellos el analfabetismo del $97.7 \%$ de la comunidad, el nivel irrisorio de la asistencia médica, que marcaba casos de $50 \%$ de muertes por enfermedades infecciosas prevenibles, como tuberculosis pulmonar, entre otras. Por solo citar algunos ejemplos de la composición histórica de las personas residentes en las zonas costeras de Angola (Monal Aleida 1974).

El cuadro actual aunque algo mejorado, no es muy diferente se compone de familias independientes, que se asientan en diferentes formas de estructura social, que sufren la falta de agua potable, de urbanización y de infraestructuras modernas que desarrollen el país.

\section{1- Principales problemas ambientales que enfrentan las comunidades costeras angoleñas.}

Los problemas medioambientales constituyen, problemas reales, tangibles o intangibles, de origen natural o derivados de la actividad humana, que ocasionan incomodidades al ser humano por el menoscabo de un bien jurídico ambiental que puede ubicarse en la naturaleza, las construcciones humanas del entorno social o el valor histórico cultural que ha adquirido por la significación subjetiva que un grupo social determinado a configurado en torno a ese bien jurídico ${ }^{4}$.

\footnotetext{
${ }^{4}$ Dra. Díaz Rodríguez, Santa Nurkis. Tipos de problemas medioambientales. Disponible en CD. Memorias del Congreso Internacional JURISCUBA 2011.
} 
Dentro de los principales problemas ambientales que persisten en el medio ambiente humano ${ }^{5}$ de las costas de Angola, se encuentran: La contaminación ambiental en sus variantes de contaminación atmosférica, sónica, de las aguas, del suelo; la acumulación de desechos; la pérdida de diversidad biológica; la degradación de los ecosistemas costeros determinada por la pesca excesiva e irracional y el desarrollo no planificado ni organizado de la acuicultura que tiene su máxima expresión en la degradación de los arrecifes coralinos, manglares, estuarios y tierras pantanosas.

El diagnóstico fáctico efectuado en la zona costera de Luanda (capital de Angola), arrojó como resultado además, que estos problemas ambientales tienen una causa común derivada de la actividad humana: la pesca ilegal en el litoral angoleño que genera:

- Presencia continua de conflictos asociados al manejo sectorial de los recursos naturales en la franja marítimo-costera de Angola.

- Dispersión en la aplicación de los instrumentos de la gestión ambiental que dan una perspectiva de gobernabilidad y seguridad jurídica.

- Aumento significativo de los actos ilícitos ambientales que se suscitan en esta zona.

- Desarrollo del país de forma no sostenible.

- Asignación poco equitativa de los recursos naturales en perjuicio de la comunidad.

Particular que ampara la necesidad de intervenir en la transformación socioambiental del sujeto cognoscente ambiental desde la transdisciplinariedad.

\section{4.- La necesidad social de la intervención universitaria en los procesos medioambientales en las comunidades costeras de Angola desde la pedagogía.}

La pedagogía como ciencia aporta las herramientas metodológicas para la formación de este estilo de pensamiento a partir de las prácticas inclusivas de pedagogía social que descansan en la ayuda que se ofrece a quienes están en alto riesgo social que comprende la reinserción ante conductas desviadas en relación al medio ambiente a partir del empleo de las vías no formales de la educación para el caso de la transformación del estilo de pensamiento de la comunidad.

Sin desconocer la labor que se realiza en la vía formal como parte del proceso docente educativo en la carrera de Derecho desde donde la didáctica pedagógica solventa las falencias del currículo universitario, convirtiendo al profesional del Derecho en un gestor de los procesos ambientales. Entendidos estos como el conjunto de acciones estructuradas de forma natural, por la

\footnotetext{
${ }^{5}$ Entendiéndose como Medio Humano al caracterizado por la escaza variedad de especies biológicas, precisamente por ser el medio donde prevalece una sola especie, la humana pero bajo la fragmentación construida socialmente de razas, civilizaciones y culturas.
} 
acción del hombre o combinando ambas, que impactan negativa o favorablemente el medio ambiente ${ }^{6}$.

Desde esta perspectiva, la universidad recupera su papel de interface como mediadora de procesos sociales en los que la ciencia, la tecnología y la acción del hombre impactan el medio natural que comparten como bien común.

Coincidiendo con la percepción de Pedagogía Social como aquel proceso a través del cual se forma al hombre como ciudadano rescatando los valores morales que son parte de su género y se le proporciona la posibilidad de integrarse cultural y socialmente a la vida de la comunidad, esto se señala con el Sistema de Procedimientos Metodológicos para la Formación del Pensamiento, con mayor énfasis en el principio que acata la educación que no es otro que el de la "socialización"?

En consecuencia esa pedagogía inclusiva, democratizadora parte de la aplicación en la educación como mediadora de procesos ambientales de las siguientes reglas:

La legitimación de la universidad como gestor de procesos de transformación sociocultural, tomando como sustento la extensión universitaria.

- Una concepción de la educación, el conocimiento y el aprendizaje que colocan neurálgicamente al estudiante como individuo social responsable, reflexivo y transformador de la realidad social circundante.

- La potenciación de las capacidades del pensamiento de más alto orden y el despliegue de la creatividad integral de los agentes participantes: profesores, orientadores, estudiantes y sujeto aprehendiente.

- La humanización de la responsabilidad ambiental como cualidad inherente al ser humano.

- La accesibilidad al conocimiento científico a partir de la capacitación a la comunidad por las vías formales e informales.

Lógicamente estas reglas por sus implicaciones resuelven determinadas contradicciones sociales y generan otras, las que se entraran a resolver en la medida de que el sistema transforme mediante acciones sociales el entorno.

\section{1 - Contradicciones sociales que se proponen resolver:}

1. La existente entre las políticas ambientales y educacionales en relación con la situación ambiental angoleña.

2. La contradicción entre la intencionalidad y la dirección del proceso docente educativo.

\footnotetext{
${ }^{6}$ Díaz Rodríguez, Santa Nurkis: Insuficiencias del currículo universitario del profesional del Derecho. En Sistema de Procedimientos Metodológicos para la Formación del Pensamiento lusfilosófico. Tesis doctoral. Centro de estudio "Manuel F. Gran", UO. Santiago de Cuba.2011, p 111.

${ }^{7}$ Dr. Ovidio D’Angelo Hernández: "La instrumentación práctica del enfoque de desarrollo humano reflexivo-creativo en el contexto social. Experiencias y perspectivas". disponible en http://www.clacso.edu.ar
} 
3. La suscitada entre los esquemas o paradigmas que modelan al profesional y la responsabilidad como ciudadano ambiental.

Es significativo aclarar que con la salida de esta investigación solo se resuelven Contradicciones Sociales desde la pedagogía, por ser la esfera específica del conocimiento desde donde se propone modelar la dinámica de la formación del pensamiento ambiental como estilo de pensamiento científico. Por lo que quedarán otras por resolver por parte de la Administración Pública, si tenemos en cuenta que la educación es un servicio público que ofrece esta administración hacia sus administrados.

\section{2.- Contradicciones sociales generadas.}

1. Entre lo tradicional y lo que se pretende generalizar como nueva estrategia de formación, manifestada en la resistencia al cambio.

2. Entre los intereses económicos y los intereses ambientales de la comunidad.

3. Entre la diversidad de los procesos ambientales y la singularidad que engendra la propuesta.

4. Entre el paradigma del consumo y el paradigma del compromiso ambiental.

\section{3.- La necesidad social de la intervención universitaria en los procesos medioambientales en las comunidades costeras de Angola desde el Derecho.}

Para explicar la ontología del pensamiento ambiental como función intelectual cosmovisiva en relación a la actividad humana como elemento trascendente de los problemas y procesos ambientales, recurrimos a la responsabilidad como categoría que determina ese estilo de pensamiento y la responsabilidad como ha sido apreciada hasta el día de hoy, es una categoría jurídica.

Según Wikipedia ${ }^{8}$, la enciclopedia libre; la responsabilidad jurídica es la imputabilidad jurídica de un hecho jurídico causada por la culpabilidad (dolosa o no) de la persona o por el simple acaecimiento del hecho desligado de la culpabilidad (responsabilidad objetiva); que supone el nacimiento de obligaciones para el imputado, y el nacimiento de derechos para el sujeto que se encuentre en posición de reclamarlas.

En el ámbito penal, la responsabilidad parte de este mismo supuesto, aunque la evolución histórica de la disciplina ha excluido la existencia de responsabilidad penal objetiva (se requiere culpabilidad) y se ha distanciado de las consecuencias antes descritas, limitándose a una sanción cuyos fines no son indemnizatorios, sino preventivos. Donde la imputabilidad surge de la existencia de una decisión de autoridad que atribuye tal condición por la transgresión de un deber ser (obligación o prohibición), o bien por otras razones justificadas en la conveniencia social de que una determinada persona no culpable sea responsable objetivamente.

Distinguiéndose jurídicamente entre responsabilidad administrativa ambiental, civil ambiental y penal ambiental.

\footnotetext{
${ }^{8}$ Búsqueda del 18 de Julio de 2011.
} 
Identificándose la responsabilidad ambiental con aquellas conductas que son realizadas por los servidores públicos, concesionarios de un servicio ambiental, o particulares, con las cuales provocan un daño al medio ambiente o a sus ecosistemas y que en dependencia de la naturaleza de la infracción sea entendida como una contravención un ilícito penal o una responsabilidad civil de reparación de daños.

La ciencia del Derecho puede aportar a la solución del problema social, el cambio conceptual de la categoría trascendente al modelo de formación del pensamiento ambiental como estilo de pensamiento científico habida cuenta que hasta hoy es entendida como una categoría jurídica preferentemente.

Díaz (2011) ${ }^{9}$, advierte que la responsabilidad, requiere la contemplación desde una apreciación cosmovisiva en espacio-tiempo, lo que implica que el profesional del Derecho acumule un contenido cultural, epistémico-jurídico que le facilite la aplicación del conocimiento jurídico, con los métodos científicos específicos de la ciencia jurídica en relación con otras ciencias que poseen contenidos vinculantes.

Connotando finalmente una sucesión de indeterminados actos conscientes de ejercicio de la voluntad desde la libertad personal del sujeto que actúa, en una dinámica entre la voluntad de adoptar una decisión en ejercicio de la libertad intelectual, desde el punto de vista ambiental.

En ese orden es criterio de los autores de la presente investigación que la responsabilidad ambiental constituye un valor configurable, transformador de un esquema cognitivo en relación al medio ambiente que trasciende las fronteras del Derecho habida cuenta que la modelación de la dinámica de su formación recae en el terreno de otras ciencias.

Desde la ciencia del Derecho, la propuesta debe observar las siguientes normas:

- Observancia de la categoría: Responsabilidad ambiental como una cualidad positiva (valor) inherente al ser humano, más allá o incluso anterior a la existencia de la norma jurídica.

- El reconocimiento de la labor profesional del profesional del Derecho como gestor de los procesos de legalidad ambiental.

- La concepción del Derecho Ambiental como rama del Derecho que rige toda la actividad humana, manifestante de una conducta (tácita o expresa); regida por normas (morales o escritas) en relación con el medio ambiente y que evidencia una cualidad axiológica común.

\section{Contradicciones sociales generadas.}

- La que se da entre ontología de la responsabilidad ambiental y el contenido que trasmite esta categoría.

\footnotetext{
${ }^{9}$ Díaz Rodríguez, Santa Nurkis: De la reflexión iusfilosófica como método para el aprendizaje del derecho, a la naturaleza jurídica del derecho ambiental. Disponible en www.Medioambiente.cu/Revista Cubana de Derecho Ambiental.
} 
- La que se genera entre la comprensión del carácter globalizante del Derecho Ambiental y la fragmentación normativa.

- La que se manifiesta entre el reconocimiento constitucional del derecho a un medio ambiente sano y la poca seguridad jurídica derivada de la deficiente gobernabilidad ambiental de las zona costeras de Angola.

\section{4.- La necesidad social de la intervención universitaria en los procesos medioambientales en las comunidades costeras de Angola desde la filosofía.}

Según reza en la Santa Biblia, como primera fuente de explicación de los fenómenos y su origen, después de creado el hombre a imagen y semejanza de Dios, este los bendijo y les dijo: "Fructificad y multiplicaos; Ilenad la tierra, $y$ sometedla; ejerced potestad sobre los peces del mar, las aves de los cielos y todas las bestias que se mueven sobre la tierra."

Probablemente ese fue el comienzo de la visión antropocéntrica del medioambiente que arrastramos hasta hoy y el origen de tantísimos problemas ambientales amparados en una apreciación filosóficamente equivocada del medio ambiente.

Vega Peña ${ }^{11}$ establece un paralelo entre el enfoque humano centrista de la ciencia y el medio ambiente y considera que el racionalismo de Descartes, en su legado más prolijo "El Recurso del Método", promueve el gran problema filosófico prevalente por más de 150 años posteriores: la separación del alma y del cuerpo, del espíritu y la materia.

Particular que le atribuye la cualidad de existencia exclusivamente a los seres pensantes, ya que el pensamiento le era consustancial al alma la que no puede dividirse en partes por ser exclusivamente consciente.

La materia, sin embargo, sólo tiene extensión, ocupa lugar en el espacio y siempre puede dividirse en partes cada vez más pequeñas, pero no es consciente, por consiguiente, los animales pertenecen plenamente a la realidad extensa, su vida y sus movimientos se realizan mecánicamente.

Descartes consideró a los animales como una especie de autómatas complejos. En cuanto a la realidad extensa se concibe así, un concepto totalmente mecanicista de la realidad. $Y$ sean entonces irracionalmente esclavizados bajo regímenes de consumo y utilidad.

Las corrientes pragmáticas del siglo pasado, vinieron acompañadas de la utilidad como criterio de lo moralmente justo y socialmente viable, o sea si es necesario, puedo hacerlo, no interesa a que costo, sin compromiso con el entorno y menos aún con las generaciones futuras o con el planeta en su integridad física.

\footnotetext{
${ }^{10}$ Génesis, (1; 28.) Santa Biblia. Reina-Valera.1995.

${ }^{11}$ Vega Peña, Yoandy: Investigador del Ministerio de Ciencia, Tecnología y Medio Ambiente de Cuba. Artículo presentado en la UNJC de Granma en el Segmento Regional de ECOIURE 2011.
} 
Aún la filosofía marxista, a la que nos afiliamos como sistema filosófico considera el medio ambiente como los elementos bióticos y abióticos que el hombre emplea para satisfacer sus necesidades crecientes.

En fin que a través de la historia de la filosofía que es la historia del pensamiento filosófico de los hombres, los problemas socio-ambientales han estado justificados por la satisfacción personal de la especie "más evolucionada", no obstante es justamente la filosofía la transformadora del paradigma sustentador del pensamiento limitado del hombre como depredador de su propio hábitat.

No obstante a partir de de la obra de $\mathrm{Kuhn}^{12}$ para la filosofía, la ciencia queda definida desde una perspectiva epistemológica y esta se entiende como interesada en el espacio que se mueve entre la verdad y el error, sitio donde impera una racionalidad gobernada por cánones universales que se desentiende de condicionantes sociales. He ahí el aporte que la filosofía puede dar a la propuesta, el método científico sobre el que debe erigirse.

\section{Contradicciones sociales generadas:}

- La que se establece entre la universalidad del pensamiento ambiental y lo particular contextualizado en el estilo de pensamiento que se pretende formar.

- La que se establece entre la ontología de la categoría Responsabilidad y la aplicación práctica del término.

\section{5.- La necesidad social de la intervención universitaria en los procesos medioambientales en las comunidades costeras de Angola desde la antropología.}

La antropología social y cultural como ramas de la antropología que estudian la sociedad y la cultura, es medular en la instrumentación de la propuesta, ya que la formación del pensamiento ambiental transcurriría bajo la influencia de una estrategia de intervención comunitaria que parte de un ejercicio de dirección estratégico sobre las potencialidades y limitaciones del sujeto cognoscente por medio del conocimiento de sus costumbres, relaciones parentales, estructuras políticas y económicas, urbanismo, medios de alimentación, salubridad, mitos, creencias y relaciones de los grupos humanos con el ecosistema.

Partiendo de la idea de E.B. Tylor: «Civilización o cultura es esa totalidad compleja que incluye conocimiento, creencias, arte, derecho, costumbres y cualesquiera otras actitudes o hábitos adquiridos por el ser humano como miembro de la sociedad $\gg{ }^{13}$.

La cultura es un sistema estructurado de saberes adquiridos a partir de la relación dialéctica entre la apropiación y la aprehensión de elementos ajenos que incorporados intersubjetivamente forman una totalidad, la totalidad de la que emerge según Fuentes $(2010)^{14}$ una cualidad de orden superior.

\footnotetext{
${ }^{12}$ T.S. Kuhn: La estructura de las revoluciones científicas. 1962.

${ }^{13}$ Concepto antropológico de cultura. Disponible en ECURED.

${ }^{14}$ Dr. C Homero Calixto Fuentes. Teoría holística sobre la configuración de la cultura.
} 
Consecuentemente la totalidad es una propiedad inherente de los sistemas donde sus partes integrantes están interrelacionadas de tal forma que un cambio en una de ellas provoca un cambio en todas las demás y en la cualidad de orden superior que lo tipifica.

En definitivas la antropología cultural aporta a la solución del problema CTS declarado la visión sobre aquellos elementos culturales, normas morales, tradiciones, religiosidad, estructura social, intereses, compromisos, aspiraciones, incluidos los recursos técnicos y económicos utilizados frente al medio natural, los modos de relación con otros hombres y hacia el manejo del patrimonio natural, histórico y cultural de la comunidad así como las motivaciones y realizaciones del grupo social seleccionado para implementar los resultados de la investigación que ampara este ejercicio.

Tecnología Educativa Propuesta. Se propone como tecnología educativa una Estrategia de Intervención Comunitaria para la Formación del Pensamiento Ambiental sustentada en un modelo didáctico transdiciplinario que favorezca la responsabilidad socio-profesional ambiental del ciudadano angoleño.

Como hemos venido señalando, esta estrategia se desarrollaría como parte de la labor extensionista de la universidad y estaría encaminada a favorecer los procesos de configuración de la cultura fuera de las aulas universitarias, dando una perspectiva realista de los problemas ambientales y llevando nuevos conceptos a sectores marginados de la población.

Se emplearían como medios para la implementación de la estrategia: un sistema de cuadernos prácticos estructurados de conformidad con los programas docentes de primero a quinto año, permitiendo la sostenibilidad y permanencia de la intervención comunitaria desde la carrera de Derecho en Angola.

Estos cuadernos dirigen el proceso docente orientándole al estudiante aquellas invariantes de conocimiento que deben tratar en la relación orientacióncomprensión del carácter globalizante de los problemas ambientales en la zona costera.

La orientación comprensiva es un vínculo interpersonal, donde se movilizan en función del crecimiento socio-profesional- ambiental de los estudiantes y la comunidad en su unidad y diferencias, los recursos personales direccionales del profesor en un contexto educativo que lo facilita. Es poner al estudiante en función de aprehender, crecer y resolver de forma desarrolladora los problemas ambientales comunitarios relacionados con el ejercicio de la profesión, lograr que cada decisión y actuación del alumno estimule el desarrollo de sus motivos, sentimientos y valores.

En tanto la comprensión está relacionada con la forma de entender la racionalidad de la acción. "Comprender" es acceder a una forma de pensamiento totalizadora desde los límites de la cultura del que comprende, los límites de la comprensión serán también los límites de la cultura a la cual se pertenece. 


\section{Resultados esperados.}

Los resultados podemos clasificarlos en resultados a mediano y largo alcance, los resultados a mediano alcance descansan en el perfeccionamiento del proceso docente educativo a partir de la inclusión social en la labor de extensión universitaria.

Revelar la lógica de la formación del pensamiento ambiental como vía de solución de los problemas ambientales asociados al empleo de la ciencia y la tecnología y aportar un método que revoluciona la enseñanza del Derecho expresando la esencia de la construcción hermenéutica reflexiva en la formación de profesional.

Como resultado a largo plazo: el desarrollo sostenible que implica la capacidad de la comunidad humana costera de Luanda de apoyar en su medio ambiente el mejoramiento continuo de la calidad de vida de sus miembros, significando sobre todo una transformación en los "modelos mentales", que permitan un cambio de percepción donde se torne más clara la dependencia que el hombre tiene del medio ambiente, se dé por sentado que en la simiente de un problema jurídico ambiental existe una concepción equivocada de medio ambiente y disminuyan en definitivas los problemas ambientales descritos en el cuerpo de ésta investigación.

\section{5.- Conclusiones:}

Haber realizado el análisis teórico de los referentes gnoseológicos de los estudios Ciencia, la Tecnología y la Sociedad, los que nos permitió revelar que estos en Cuba han dejado la vía expedita para la instrumentación de propuestas como esta, generalizables en otros países con características similares.

La necesidad de perfección del proceso docente educativo y distinguir las debilidades en la formación del profesional del Derecho desde diferentes ciencias lo que facilitó el diagnóstico del saber hacer de los estudiantes.

El Estado y Gobierno angoleño aboga por un desarrollo socio-profesionalambiental de la mayoría de su sufrida población, donde, sus reservas naturales constituyen una fuente importante a explotar, pero no ha logrado implementar las políticas legales en ese sentido.

El análisis por parte del estado angoleño a que se dicte una normativa especial en relación con la responsabilidad ambiental, en pos de la mitigación de los problemas ambientales, delimitando el fuero judicial donde tendrán la debida respuesta estos conflictos ambientales que en el mismo sean conocidos.

El Estado deberá promover campañas de bien público para fomentar la cultura por parte de la población en preservar el medio ambiente, que partan desde la formación académica en todos los niveles de la enseñanza en Angola. 
Que se realicen estudios jurídicos para modernizar la actual legislación marco ambiental, para esto podrán contar con la experticia de juristas de las Universidades angoleñas y de los operadores del derecho que prestan sus servicios de asistencia legal en instituciones estatales y privadas en el país.

\section{6.- Bibliografía}

ANTÚNEZ SÁNCHEZ, A. (2009)Dimensión social-jurídica del medio ambiente. Una apreciación de la problemática ambiental..

ANTÚNEZ SÁNCHEZ, A. Y BALLESTER NÁPOLES, L. (2009) Los problemas Medio Ambientales que afectan la actividad pesquera en el Golfo del Guacanayabo, municipio Manzanillo, Provincia Granma. Tesis en opción al título de Máster en Derecho Ambiental..

BASAVÉ FERNÁNDEZ DEL VALLE, A. (2001): Filosofía del Derecho Internacional, Universidad Nacional Autónoma de México,.

BECERRA RAMÍREZ, M. (2007): Derecho Internacional Público, Biblioteca Jurídica Virtual de la UNAM, IIJ-UNAM, México, D. F.,.

BRAÑES, R.: “Manual de Derecho Ambiental”, Fundación Mexicana para la Educación Ambiental, fondo de Cultura Económica, 1ra. Edición, México.

CANOVAS GONZALEZ, Dr. C. Daimar. (2011) Estudios sobre Derecho del Ambiente. Editora Vadell hermanos. Valencia. Venezuela.

CAÑIZARES ABELEDO, F. (1979) “Teoría General de Estado y del Derecho”.

CARABALLO MAQUEIRA, L. (2000) Derecho Ambiental cubano. La Habana.

CARRILlO SALCEDO, J. A. (1994) Curso de Derecho Internacional Público. Introducción a su estructura, dinámica y funciones, Editorial Tecnos S. A, 1ra. Edición, 2da. Reimpresión, Madrid.

COLECTIVO DE AUTORES (2007) Derecho Ambiental Cubano, Editorial Félix Varela. La Habana.

COLECTIVO DE AUTORES (2005) Introducción al conocimiento del medio ambiente (Suplemento Especial). Universidad para Todos. CITMA-CIGEA, La Habana, p. 3.

CONFERENCIA SOBRE EL MEDIO AMBIENTE Y DESARROLLO DE LAS NACIONES UNIDAS, Conocida Como Cumbre De La Tierra, Junio De 1992, Río De Janeiro, Brasil.

D’ ESTÉFANO PISANI, M. A (2003) Breve historia del Derecho Internacional: desde la Antigüedad hasta el año 2002, Editorial de Ciencias Sociales, La Habana.

: ENCICLOPEDIA MICROSOFT ENCARTA ONLINE: Angola.

Microsoft Corporation. Disponible en:

http://es.encarta.msn.com (C) Derechos Reservados, 1997-2009

: Esquemas del Derecho Internacional Público, Tomo I, Editorial Pueblo y

Educación, La Habana, 1980.

DÍAZ, DELEGADO, J. CARLOS (1999): Ecología y Sociedad, Estudios editorial Ciencias Sociales, La Habana.

DÍAZ RODRÍGUEZ, Dra.C. SANTA NURKIS (2011) Insuficiencias del currículo universitario del profesional del Derecho. En Sistema de Procedimientos Metodológicos para la Formación del Pensamiento Iusfilosófico. Tesis doctoral. Centro de estudio "Manuel F. Gran", UO. Santiago de Cuba.111p.

DIEZ DE VELASCO, M (2005): Instituciones de Derecho Internacional Público, 15ta. Edición, Editorial Tecnos, Madrid. 
: Diccionario Pequeño Larousse ilustrado Ministerio de Educación Edición Revolucionaria 1968. P 1995.

: Disposiciones jurídicas relacionadas con el Derecho de Tratados en materia de medio ambiente. Libro electrónico "Ciencias de la tierra y del medio ambiente".

DE AHUMADA RAMOS, FRANCISCO JAVIER: Las formas de actuación de las administraciones pública. En: http://www.org/dewalac/esp/.

DIEZ DE VELASCA, M. (2005) Instituciones de Derecho Internacional Público, 15ta. Edición, Editorial Tecnos, Madrid.

: Instituciones de Derecho Internacional Público (en tres Tomos),

s/a.

: Las organizaciones internacionales, 10ma. Edición, Editorial

Tecnos, Madrid, 1997.

DICCIONARIO DE TÉRMINOS AMBIENTALES (1998) Publicaciones Acuario, Centro Félix Varela. Camacho Barreiro, A y L. Ariosa Roche. La Habana. Cuba..

ENGELS, Federico (1979). Introducción a la dialéctica de la naturaleza. Obras Escogidas en dos tomos. Tomo 2. Editorial Ciencias Sociales. La Habana. Cuba.

ELIAS VEGA, Niobis. El manejo integrado de zonas costeras. Tesis en opción al título de Especialista en Derecho de Empresas. Facultad de Derecho. Universidad de Oriente. Cuba.

FERNÁNDEZ-RUBIO LEGRÁ, A (1991): Instrumentos Jurídicos Internacionales, Editorial Pueblo y Educación, La Habana, Cuba, Tomo II. : Derecho Ambiental Internacional, La Habana, Cuba, 1996.

FUENTES COBAS, Milagros (2013). La responsabilidad ambiental dentro del ordenamiento jurídico, visión cubana. Tesis en opción al título de Licenciado en Derecho. Universidad de Granma.

GALLO, PIMENTEL JOSÉ M; GONZÁLEZ, DÍAZ ERNESTO (2000) Introducción a la Metodología de Investigación Pedagógica y Técnica. Instituto Superior Pedagógico para la Educación Técnica y Profesional. Ciudad de La Habana. Cuba. p 92.

: GESOCYT (1994). Problemas Sociales de la Ciencia y Tecnología, Editorial Félix Varela. La Habana.

GUADARRAMA, G. P.; Suárez Gómez Carmen (2000) ética, Ciencia y Tecnología. En Filosofía y Sociedad. Tomo II. Editorial Félix Varela, La Habana. p.518-523.

GOMES CANOTILHO, JOSÉ JOAQUIM (2009) Intruduçao ao Direito do Ambiente, Universidade Aberta do Lisboa, 1ra. Ediçao, Portugal.

GONZÁLEZ CAMPOS, J. D. Y SAENZ DE SANTA MARÍA, P. A (2007) Legislación básica sobre Derecho internacional público, Séptima Edición actualizada, Editorial Tecnos, Madrid.

GUADARRAMA, G. P.; SUÁREZ GÓMEZ, CARMEN (2000) Ética, Ciencia y Tecnología. En Filosofía y Sociedad. Tomo II. Editorial Félix Varela, La Habana. p.518-523

HERDEGEN, M (2007) Derecho Internacional Público, Biblioteca Jurídica Virtual de la UNAM, IIJ-UNAM, México, D. F.

HERNÁNDEZ GIL, ANTONIO: Conceptos jurídicos fundamentales, Obras completas, Editorial, Espasa Calpe, Tomo 1.

INGUNZA MILlITZA, FRANCISKOVIC: Perspectiva del Derecho Ambiental Internacional. Aspectos críticos. En (http://www.org/dewalac/esp/)10/11. 
JUSTE RUIZ, J (1998) La protección del medio ambiente en su dimensión internacional, La Habana.

KEDROV, B. M (1982): La ciencia en Historia de las ciencias e historia de la Filosofía. Editorial Pueblo y Educación. La Habana.

KALIN ARROYOS y autores (2013). Ciencias Ambientales. Diagnóstico y una mirada hacia el futuro. Chile.

KOROVIN, Y. ET AL (1963) Derecho Internacional Público, Editorial Grijalbo, S. A, México D.F.

KUN T.S (1962): La estructura de las revoluciones científicas. Editorial Academia, La Habana. Cuba..

: Ministério da Educação: Caracterização Global do Contexto Angolano e Respectivo Sistema Educativo. Reforma Curricular/INIDE/2003.p 28.

: Ministerio de Asuntos Exteriores y de Cooperación. Plan África 2009-2012. Editado por Agencia Española de Cooperación Internacional para el Desarrollo. Artículo presentado en la UNJC de Granma en el Segmento Regional de ECOIURE 2011.pág.15. www.casafrica.es/casafrica/Inicio/PlanAfrica2009-

LOZANO CUTANDA, Blanca (2004). Derecho Ambiental Administrativo, 5ta edición, Editora Dykinson, Madrid. España.

Infracciones y sanciones administrativas para la protección del medioambiente, en Alonso García, E y Lozano Cutanda, B. (2005) Diccionario de Derecho Ambiental, Editora Iustel. Madrid, España.

LIBRO VERDE LA UNIÓN EUROPEA SOBRE RESPONSABILIDAD SOCIAL DE LAS EMPRESAS. CE. 2012

MONAL, ALEIDA (1974): África a partir de la Segunda Guerra Mundial. Selección de Materiales Bibliográficos. Dirección de Producción de Medios de Enseñanza. Viceministerio de Economía y Servicios Generales. MINED. La Habana Cuba. p. 89-93.

MONCAYO, VINUESA, GUTIÉRREZ POSSE (1990) Derecho Internacional Público, Tomo I, 3ra. Reimpresión, Editorial Zavalia, Buenos Aires.

MONZON, Dra.C. Yailen (2012) Tesis doctoral El Manejo Integral de las Zonas Costeras en Cuba. Universidad de Cienfuegos. República de Cuba.

NÚÑEZ JOVER, JORGE y CASTRO DÍAZ-BALART: (2001) La ciencia y la tecnología como procesos sociales. Lo que la educación científica no debería olvidar. Editorial Juventud Rebelde. La Habana.p.10

NÚÑEZ JOVER, JORGE (2003) la ciencia y la tecnología como procesos sociales. La Habana. Cuba..

PARADA, RAMÓN (1997) Derecho Administrativo, 9na. Edición, Editorial Marcial Pons, Madrid, parte general I.

PASTOR RIDRUEJO, J. A (1992): Curso de Derecho Internacional Público y Organizaciones Internacionales, 4ta. Edición, Editorial Tecnos S. A, Madrid.

PINO CANALES, C. ET AL (2005): Temas de Derecho Internacional Público, Editorial Félix Varela, La Habana.

PODESTA COSTA, L. A Y RUDA, J. MA (1988) Derecho Internacional Público, Tomo 1, Tipográfica Editora Argentina, Buenos Aires.

PUENTE EGIDO, J (1991): Casos prácticos de Derecho Internacional Público, Dykinson, S. L., Madrid,.

: PNUMA., Serie de Documentos sobre Derecho Ambiental del Programa de Naciones Unidas para el medio ambiente, No. 3. 
RICHARTZ, SASKIA: directora de políticas oceánicas de la organización ambientalista Greenpeace.http://estasemana.cip.cu/noticias/la-pesca-ilegal-causaperdidas-millonarias-en-africa.

ROJAS AMANDI, VÍCTOR MANUEL: El Derecho Ambiental Internacional Público del medio ambiente al inicio del siglo XXI, Consultado en: http://www.fao.org/spanish/newsroom/news/2003/25379-es.html.

ROSENTAL, M. y P. IUDIN (1973) Diccionario Filosófico. Ed. Pueblo y Educación. La Habana, p.33

ROSENTAL, M. y P. IUDIN (1981) Diccionario Filosófico. Ed. Pueblo y Educación. La Habana. p.256-257.

RUBINSTEIN, L. S (1965) El ser y la conciencia. Editorial Universitaria, La Habana, 443pp.

RUTNIKAS, L. BERTHA; ORAMAS, DM; NOYA G. LISETTE et al (1991) Se aprende a conversar. Editorial Pueblo y Educación. La Habana. p 11.

SOCARRÁS STABLE, JOHANNA Y DEL CASTILLO POMARES, TAMARA: Medio Ambiente, en Aspectos Generales del medio ambiente y de la Gestión Ambiental Empresarial, Grupo de Trabajo Estatal, Bahía Habana, p.10.

SUÁREZ, CLARA (1994). La orientación educativa. Una experiencia en la educación. Folleto de psicología. CEES, "Manuel F. Gran" UO, p 99.

TUNKIN, G (1979) Curso de Derecho Internacional, Libros 1y 2, Editorial Progreso, Moscú.

: UNESPA (2000) Estudio del nivel de dependencia, necesidades de recursos y proyecciones de futuro.

: UNIÓN EUROPEA (1997) comunicado de la Comisión, de 12 de marzo de: Política Social y Protección Social. Modernización y mejora de la protección. 1998.

VALVERDE, M.D.; FLÓREZ, M., Y SÁNCHEZ, I (2001) Escalas de actividades de la vida diaria. Colección Rehabilitación, 28. IMSERSO.

VERDUGO, M.A., URRIES, J (2001) Apoyos, autodeterminación y calidad de vida. Salamanca, Amaru.

VIAMONTES GUILBEAUX, Dra.C. EULALIA (2007) Derecho Ambiental Cubano, La Habana, Editorial Félix Varela.

: La responsabilidad administrativa ambiental (2006) Proyecto Ciudadanía Ambiental y CIGEA., Multimedia Derecho Ambiental Cubano, La Habana

VICTOR JOAO DOMINGOS; y autores (2012) La pesca ilícita en el ámbito del Derecho Ambiental Internacional. Situación en Angola. Trabajo de Diploma. Universidad de Granma. Cuba. p 74.

ZELEDÓN ZELEDÓN, Dr. Ricardo (1998). Código Ambiental, Editora Porvenir, Primera Edición. San José. Costa Rica. 